\title{
Therapie mit Antiepileptika verlängert nicht das Überleben
}

Fragestellung: Einige retrospektive Analysen und eine ungeplante Post-hoc-Analyse der Zulassungsstudie für Temozolomid [1] hatten die Vermutung begründet, dass die Gabe bestimmter Antiepileptika, hier vor allem Valproat (VPA) oder Levetiracetam (LEV), das Überleben von Glioblastompatienten verlängern könnte. Verschiedene Mechanismen wurden hierfür postuliert, zum Beispiel die enzymhemmende Wirkung von VPA mit konsekutiver Erhöhung der Serumkonzentration von Temozolomid oder dessen direkte tumorizide Wirkung durch die Wirkung als Histon-Deacetylase [2].

Patienten und Methodik: Aus der oben zitierten Studie $(\mathrm{n}=287)$ und insgesamt vier weiteren prospektiven, randomisierten Therapiestudien zur Primärtherapie des Glioblastoms (Validierungsgruppe) wurden Daten von insgesamt 1.869 Patienten gepoolt und ausgewertet. Dabei handelte es sich um solche Patienten in den randomisierten Studien, die im Kontrollarm die Standardtherapie mit konkomitanter Radio- und TemozolomidChemotherapie sowie nachfolgender adjuvanter Temo-

Happold C, Gorlia T, Chinot O et al. Does valproic acid or levetiracetam improve survival in glioblastoma? A pooled analysis of prospective clinical trials in newly diagnosed glioblastoma. J Clin Oncol 2016; 34 : $731-9$ zolomid-Therapie erhalten hatten, oder um Patienten aus den experimentellen Armen, bei denen die zu prüfende zusätzliche Substanz (Cilengitide) keinen Überlebensvorteil erbrachte. Diese Daten wurden für Gesamt- überleben und progressionsfreie Überlebenszeit ausgewertet und einer Cox-Regression unterworfen, in die nach Studie stratifiziert und nach prognostischen Faktoren adjustiert wurde. Ausgehend von der Post-hoc-Analyse [2] der EORTC-Zulassungsstudie für Temozolomid [1] wurden Patienten, die den Enzymhemmer VPA bekommen hatten, mit Patienten verglichen, die entweder gar keine Antiepileptika, enzyminduzierende Antiepileptika oder nicht enzyminduzierende Antiepileptika erhalten hatten. Dieser Vergleich wurde für Patienten durchgeführt, die zu irgendeinem Zeitpunkt VPA eingenommen hatten und dann auch zusätzlich für Patienten, die über den Zeitraum der tumorspezifischen Therapie hinaus VPA verabreicht bekamen. Eine entsprechende Analyse erfolgte auch für LEV.

Ergebnisse: In der unifaktoriellen Analyse erwiesen sich die bekannten prognostischen Faktoren als Einflussgrößen auf progressionsfreies und Gesamtüberleben. So hatten junges Lebensalter, positiver MGMT-Promotor-Methylierungsstatus und guter klinischer Zustand einen positiven Einfluss. Dagegen zeigten ausnahmslos alle Analysen der verschiedenen Gruppen und Untergruppen keinen Einfluss der Einnahme von VPA (zu irgendeinem Zeitpunkt oder langfristig gegeben) auf progressionsfreies oder Gesamtüberleben. Das traf auch für LEV zu.

Schlussfolgerungen: Die Autoren schließen aus den vorgestellten Daten, dass Antiepileptika keinen unmittelbaren Einfluss auf das progressionsfreie oder Gesamtüberleben von Glioblastompatienten haben.

\section{- Kommentar von Uwe Schlegel, Bochum}

\section{Die Hoffnung auf eine Prognoseverbesserung hat sich nicht erfüllt}

Der hier vorliegende umfassende und vorbildlich aufgearbeitete Datensatz ist von erheblicher Bedeutung für die klinische Neurologie. Insbesondere die 2011 vorlegte ungeplante Posthoc-Analyse an 287 Patienten aus der Temozolomid-Zulassungsstudie [2] hatte annehmen lassen, dass für die Patienten, die Temozolomid erhalten, der Enzymhemmer VPA mit einer Verlängerung von progressionsfreiem und Gesamtüberleben verbunden ist. Das schien insbesondere vor dem Hintergrund plausibel, dass eine effektive Enzymhemmung den Metabolismus von Temozolomid reduzieren und damit dessen Bioverfügbarkeit erhöhen würde. Die jetzt vorliegenden Daten belegen eindrucksvoll, dass sich eine solche Schlussfolgerung nicht ziehen lässt. Für die klinische Neurologie bedeutet das, dass Glioblastompatienten nur dann mit Antiepileptika behandelt werden sollten, wenn eine symptomatische Epilepsie dieses erforderlich erscheinen lässt. Hoffnungen auf einen unmittelbaren positiven Einfluss auf die Prognose durch die Gabe von alten oder neuen Antiepileptika haben sich mit dieser Analyse nicht erfüllt.
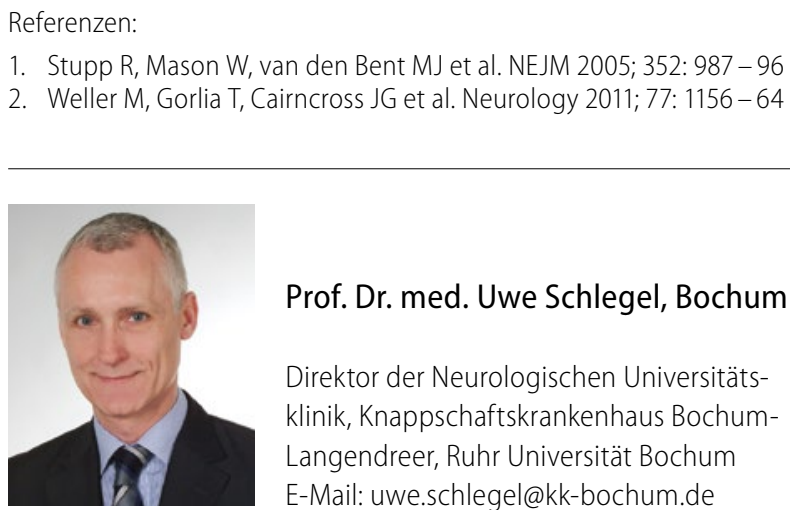

Prof. Dr. med. Uwe Schlegel, Bochum

Direktor der Neurologischen Universitätsklinik, Knappschaftskrankenhaus BochumLangendreer, Ruhr Universität Bochum E-Mail: uwe.schlegel@kk-bochum.de 